\title{
DESIGNING OPTIMUM OIL THICKNESS IN ARTIFICIAL HUMAN KNEE JOINT BY SIMULATED ANNEALING
}

\author{
Hamit Saruhan \\ Mechanical Design Division \\ University of Düzce, 81620 \\ Beçi Kampüsü, Düzce, Turkey \\ hamitsaruhan@duzce.edu.tr
}

\begin{abstract}
In human knee joints, synovial fluid film covers the surface of synovium and cartilage within the joint space. Synovial joints acts as bearing in mechanical system. Although the artificial human joints differ from most mechanical bearings in their nature, they have been modeled and analyzed in terms of hydrodynamic bearing of engineering. In this study, nature inspired an algorithm, the simulated annealing (SA), is used to find minimum fluid film thickness in artificial human knee joints. It is important to optimize the film thickness of the bearing in terms of successful operation and lifetime of the implant in terms of friction and wear. The problem is complex and a time consuming process due to design variables and constraints imposed on the objective function. It is demonstrated that the SA performed and obtained convergence reliability on the optimum point.
\end{abstract}

Key Words- Simulated annealing, Synovial fluid, Film thickness, Knee joint, Optimization

\section{INTRODUCTION}

Design and development of modern mechanical components are the result of advancement of manufacturing technology and the investigations carried through in different scientific areas. The importance of tribology in engineering design is self evident since virtually all mechanical system components are involved with relative motion. Lubrication minimizes frictional resistance between bearing surfaces by keeping them apart. Most mechanical bearings lubrication is achieved by oil hydrodynamically. When the bearing material as in knee joints is soft it may deform under the hydrodynamic pressures and elastohydrodynamic lubrication will occur [1]. The knee joint differs from most bearing in mechanical systems. The knee joint is a complex interactive biomechanical system. The mechanisms of lubrication presented in human joints have been studied for many years [2]. Also there are significant advances in the artificial human knee joint design in recent years. It is important to optimize the bearing lubrication to minimize friction and wear in artificial knee joints mechanisms. Developments in computer technology have proved to be a great chance to the world of design optimization. Any efficient optimization algorithm explores to investigate new and unknown areas in search space and exploit to make use of knowledge found at point previously visited to help find better solution point. The simulated annealing (SA) can provide a remarkable balance between exploration and exploitation of the search space. 
From this point of view, this study provides use of the SA to seek a global optimum solution to problem in hand. The SA algorithm imitates the process of annealing in metals as they cool from liquid to solid states. The algorithm employs the generation of random numbers when they search for the optimum point. It does not require the evaluation of gradient of the objective function.

\section{THE SIMULATED ANNEALING}

In this section of the paper, the fundamental intuition of the SA and how it processes are given. The SA was proposed by Kirkpatrick et al. [3] to deal with complex non-linear problems. They showed the analogy between simulating the annealing of solid as proposed by Metropolis et al. [4]. The SA is an iterative improvement algorithm for a global optimization. It is inspired from thermodynamic to simulates the physical process of annealing [5] and [6] of molten metals. It obtains the minimum value of energy by simulating annealing which is a process employed to obtain a perfect crystal by gradual cooling of molten metals [7] in order to keep the system of melt in a thermodynamic equilibrium at given temperature. Thus, it exploits an analogy between the way in which a metal cools and freezes into a minimum energy crystalline structure. At high temperature, the atoms in the molten metal can move freely with respect to each other as the cooling proceeds, the atoms of metal become more ordered and the system naturally converges towards a state of minimal energy. This formation of crystal mostly depends on the cooling rate. If the metal is cooled at very fast rate, the atoms will form an irregular structure and the crystalline state may not be achieved. The simulated annealing makes use of the Metropolis et al. [4] algorithm which provides an efficient simulation according to a probabilistic criterion stated as:

$P(\Delta E)=\left(\begin{array}{ll}1, & \text { if } \Delta E<0 \\ e^{(-\Delta E / T)}, & \text { otherwise }\end{array}\right.$

Thus, if $\Delta E<0$, the probability, $P$, is one and the change - the new point- is accepted. Otherwise, the modification is accepted at some finite probability. Each set of points of all atoms of a system is scaled by its Boltzmann probability factor $e^{(-\Delta E / T k)}$ where $\Delta E$ is the change in the energy value from one point to the next, $k$ is the Boltzmann's constant and $T$ is the current temperature as a control parameter. The general procedure for employing the SA as follows; Step 1: Start with a random initial solution, $X$, and an initial temperature, $T$, which should be high enough to allow all candidate solutions to be accepted and evaluate the objective function. Step 2: Set $i=i+1$ and generate new solution $\left(X_{i}^{\text {new }}=X_{i}+r S L_{i}\right)$ where $r$ is random number and $S L_{i}$ at each move should be decreased with the reduction of temperature. Evaluate $F_{i}^{\text {new }}=F\left(X_{i}^{\text {new }}\right)$. Step 3: Choose accept or reject the move. The probability of acceptance (depending on the current temperature) if $F_{i}^{\text {new }}\left\langle F_{i-1}\right.$, go to Step 5, else accept $F_{i}$ as the new solution with probability $e^{(-\Delta E / T)}$, where $\Delta E=F_{i}^{\text {new }}-F_{i-1}$ and go to Step 4. Step 4: If $F_{i}$ was rejected in Step 3, set $F_{i}^{\text {new }}-F_{i-1}$. Go to Step 5. Step 5: If 
satisfied with the current objective function value, $F_{i}$, stop. Otherwise, adjust the temperature $\left(T^{\text {new }}=T r_{T}\right)$ where $r_{T}$ is temperature reduction rate called cooling schedule and go to Step 2. The process is done until freezing point is reached. The major advantages of the SA are an ability to avoid becoming trapped in local optimum and dealing with highly nonlinear problem with many constraints and multiple points of optimum.

\section{FORMULATION OF THE PROBLEM}

The knee joint lubricant, synovial fluid, is composed of hyaluronate, an extremely large polymerized sugar molecule, and a mixture of proteins and electrolytes [8]. Synovial fluid is non-newtonian fluid in that its viscosity decreases with increased shear rate [9],[10]. With the lubricant film thickness stepping down, the state of lubrication will undergo the following; from dynamic lubrication to elastohydrodynamic then an unknown state (thin film lubrication) further to boundary lubrication and dry friction [11]. Elastohydrodynamic lubrication is a form of fluid film lubrication where elastic deformation becomes significant in the bearing surfaces. Elastohydrodynamic lubrication normally occurs in contacts where the film thickness is in the range $10^{-7} \leq h_{\min } \leq 10^{-6}$ [12]. The elastohydrodynamic lubrication analysis has been employed to satisfy the needs in design optimization of the knee joint. The numerical solution of the isothermal elastohydrodynamic lubrication of elliptical contacts requires the simultaneous solution of the elasticity and Reynolds equations [13]. As shown in Figure 1, the contact geometry of two rollers reduced to the contact geometry as a flat and a roller surface. The most important practical aspect of the elastohydrodynamic lubrication point-contact theory [14] is the determination of the minimum film thickness within the contact. It is very important that the prediction of successful operation of moving elements is in adequate film thickness as thin and continuous [13].

The formulation of minimum fluid film thickness in artificial knee joints, which is performed in the study by [15], will be a reference for this study. The influence of the ellipticity parameter $K$, the dimensionless speed $U$, and load $W$ on the minimum film thickness was investigated. 

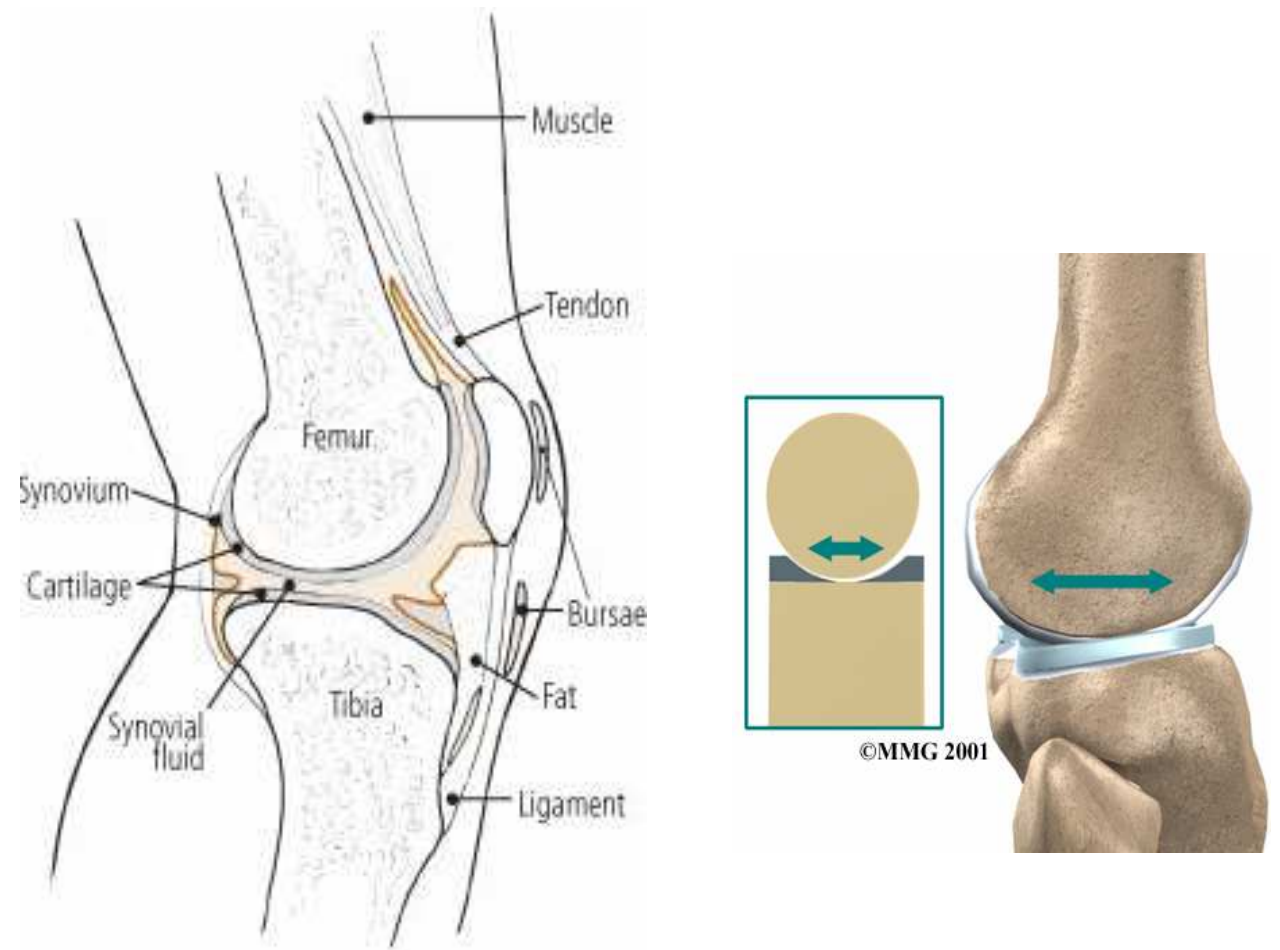

SHarriet Gręenfield

Figure 1 Human knee joint

The minimum film thickness formula based on least-squares fit of data for a fully flooded, isothermal, and elastohydrodynamic elliptical contact for low-elastic modulus materials is given as follows:

$h=7.43 U^{0.65} W^{-0.21}\left(1-0.85 e^{-0.31 K}\right)$

where $U$ is dimensionless speed parameter, $W$ is dimensionless load parameter, and $K$ is ellipticity parameter.

$U=\eta u / E^{\prime} R_{x} ; \quad W=F / E^{\prime} R_{x}^{2} ; \quad K=a / b$

where $\eta$ is absolute viscosity of synovial fluid in Pa.s. At low shear rates the viscosity of normal synovial fluid values is typically between $10^{-3}$ and $10^{-2}$ Pa.s [15]. $u$ is entraining mean velocity in $\mathrm{m} / \mathrm{s} . E^{\prime}$ is effective elastic modulus and lies in the range $10^{7}$ to $10^{9}$ in $\mathrm{Pa} . R_{x}$ is radius of equivalent sphere near a plane in $\mathrm{m}$. It ranges from 0.02 to 0.1 in the knee. $F$ is applied load in $N$. When the leg swings freely in the walking cycle, the knee carries load in the range zero to one times body weight. When the leg is in contact with the ground the load range from three to seven times the body weight [15].The load on the knee may go up to 25 times body weight in a vertical drop of one meter [16]. The ellipticity parameter, $K$, is varied from 1 to 12 for a ball on the 
plane configuration [15]. $a$ and $b$ are semi-axis of the Hertzian contact ellipse in the $x$ - and $y$-directions, respectively.

\section{EMPLOYING THE SIMULATED ANNEALING}

In the present problem it is assumed that the parameter quantities typify the typical conditions in normal walking. Thus, radius of equivalent sphere near a plane $\left(R_{x}=0.1 \mathrm{~m}\right)$, effective elastic modulus $\left(E^{\prime}=10^{9} \mathrm{~Pa}\right)$, and entraining mean velocity $(u=0.075 \mathrm{~m} / \mathrm{sn}$ ). The statement of the design of the problem is formulated as follows:

$$
h=F(\eta, F, K)=7.43\left(\eta u / E^{\prime} R_{x}\right)^{0.65}\left(F / E^{\prime} R_{x}^{2}\right)^{-0.21}\left(1-0.85 e^{-0.31 K}\right)
$$

Subject to

$$
g_{j}(\eta, F, K) \leq 0 \quad j=1, \cdots \cdots N \text { con } \quad \text { where Ncon is number of constraints. }
$$

Constraints are conditions that must be met in the optimum design and include restrictions on the design variables value and the optimum design of the objective function. These constraints define the boundaries of the feasible and infeasible design space domain. The lower and the upper limits of dimensionless speeds and loads were considered as constraints for the design problem in hand as follows:

$$
\begin{aligned}
& g_{1}=2.1410^{-12}-\left(\eta u / E^{\prime} R_{x}\right) \leq 0 \\
& g_{2}=\left(\eta u / E^{\prime} R_{x}\right)-8.9010^{-11} \leq 0 \\
& g_{3}=0.03810^{-6}-\left(F / E^{\prime} R_{x}^{2}\right) \leq 0 \\
& g_{4}=\left(F / E^{\prime} R_{x}^{2}\right)-5.3210^{-4} \leq 0
\end{aligned}
$$

Each design variable has a specified range in the following:

$$
0.001 \leq \eta \leq 0.01
$$

$450 \leq F \leq 4500$

$1 \leq K \leq 12$

The SA is unconstrained optimization procedure. Therefore, the objective function should be transformed into an unconstrained problem by setting an augmented objective function incorporating any violated constraint as penalty function. The penalized objective function, $F_{O b j}$, can be written as:

$$
F_{O b j}=F(\eta, F, K)+\sum_{j=1}^{N c o n} r_{j}\left(\max \left[0, g_{j}\right]\right)^{2}
$$


In case of any violation of a constraint boundary, the fitness of corresponding solution is penalized, and thus kept within feasible regions of the design space by increasing the value of the objective function when constraint violations are encountered. A unique static penalty function developed by Homaifar et al. [17] is used with multiple violation levels set for each constraint. Each constraint is defined by the relative degree of constraint penalty coefficient. The penalty coefficients, $r_{j}$, for the $j$ th constraints have to be judiciously selected.

By employing the SA, a random initial point is selected at high temperature and a series of moves are made according to defined annealing schedule. The change in the objective function values, $\Delta E$, is computed at each move. A new solution is generated in the neighborhood of the current configuration in each iteration. This new solution is automatically accepted with probability of 1 , if it results in decreased objective function value. Otherwise, if the new solution is increased the objective function value, the acceptance is given with a small probability, $e^{(-\Delta E / T k)}$. Where $T$ is the current temperature and $k$ is Boltzmann's constant. The probability expression suggests that if the temperature of the system is large, the probability of accepting the solution increases. Otherwise, if $T$ is low, the probability of accepting solution decreases. Therefore, the temperature needs to be high at the beginning. As the iteration proceeds, the temperature is gradually decreased until the stopping condition is met. There are many ways to determine when to stop running the algorithm: One is the temperature when reduced to a threshold. Another is to reach a pre-specified number of temperature transitions. All the generating and acceptance depend on the temperature. The global optimum can be converged by carefully controlling the rate of cooling of the temperature. The important setting parameters of the SA for this study are chosen as follows: Initial temperature $T=10000$, temperature reduction rate $r_{T}=0.5$, and number of iterations performed at a particular temperature $n=5$.

\section{RESULTS}

Figure 2, Figure 3, and Figure 4 are presented that illustrate in detail the objective function versus variables by visualizing the design space. The optimal design point which satisfies the four inequality constraints is marked on the plots. The design variables range limits divide the design space in two regions: as feasible design region in which all design constraints are satisfied, and infeasible design region in which at least one design constraint is violated. Figure 2 gives the objective function values versus the ellipticity parameter and applied load with respect to the upper and the lower limits for the absolute viscosity of fluid film. Figure 3 gives the objective function values versus the absolute viscosity of fluid film and applied load with respect to the upper and the lower limits for the ellipticity parameter. Figure 4 gives the objective function values versus the absolute viscosity of fluid film and the applied load for the optimum value of the obtained optimum ellipticity parameter held constant at $K=3.82$. Overall, the minimum film thickness of $0.9510^{-6}$ was found after 9000 function evaluations with the absolute viscosity of $4.271 \mathrm{~Pa} . \mathrm{s}$, the applied load of $4196.56 \mathrm{~N}$, and the ellipticity parameter of 3.82 . 


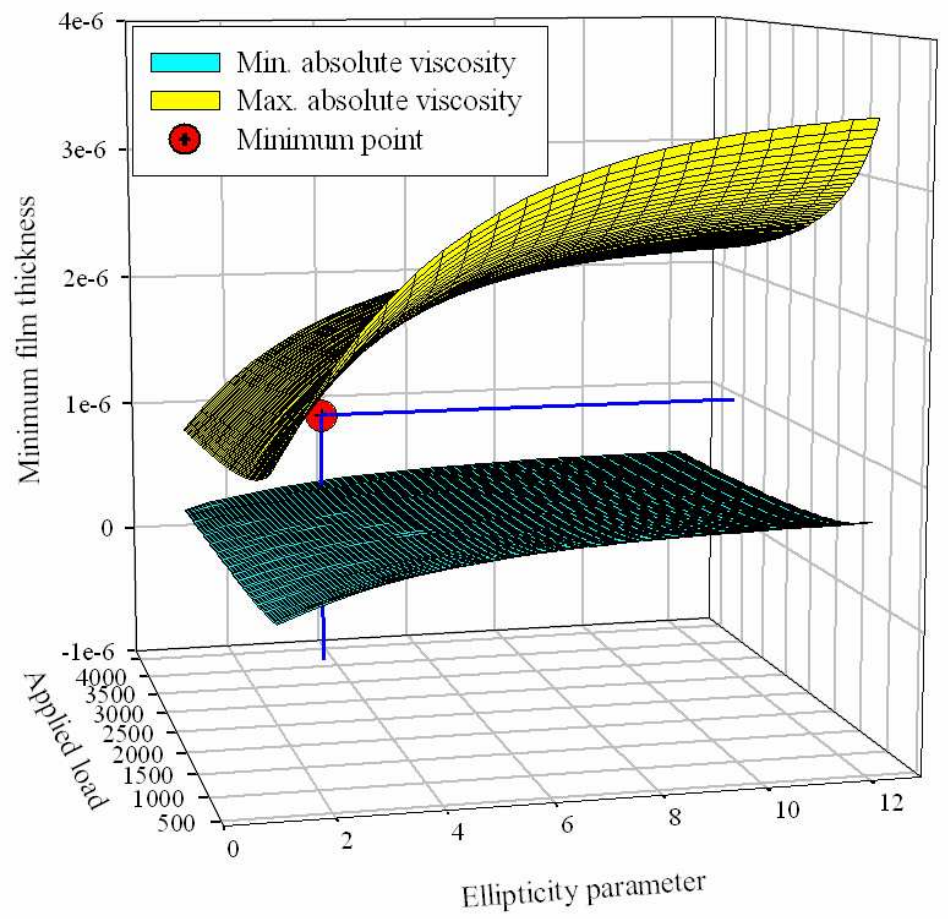

Figure 2 The objective function values versus the ellipticity parameter and applied load with respect to the upper and the lower limits for the absolute viscosity

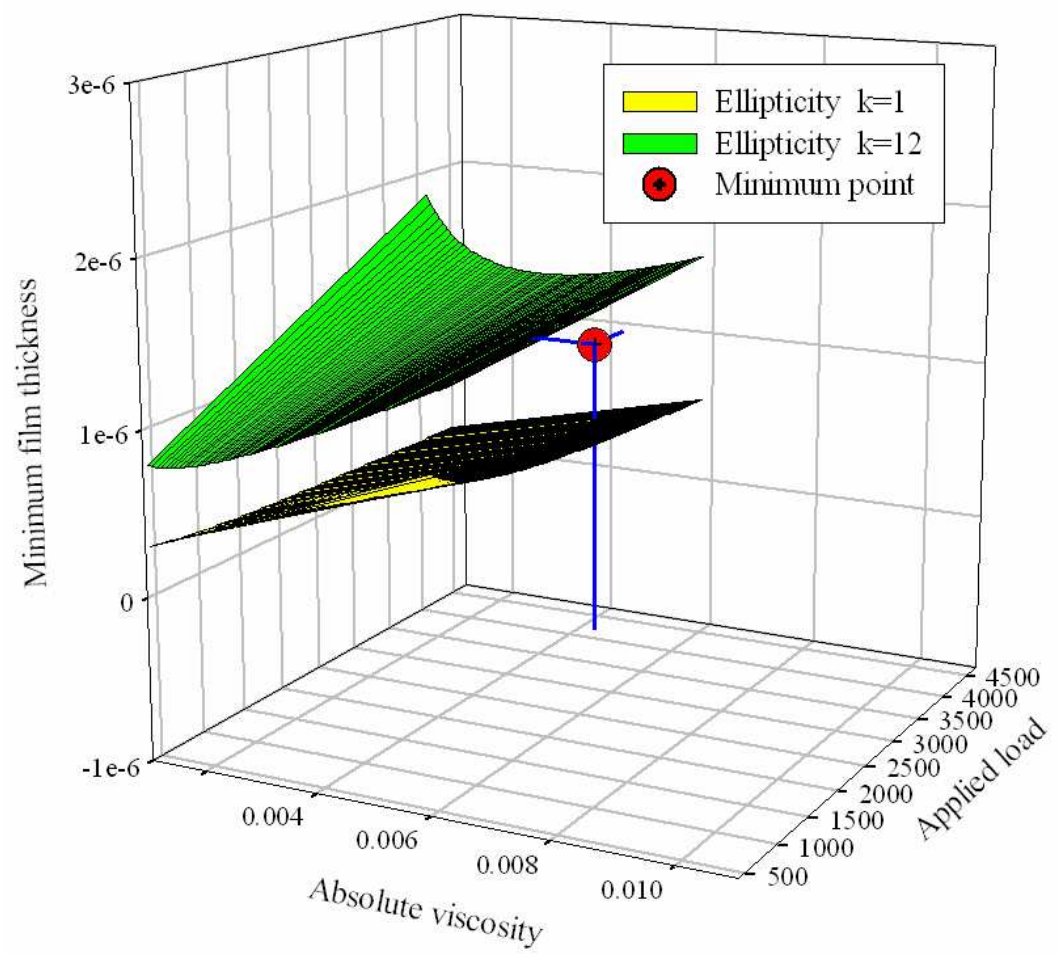

Figure 3 The objective function values versus the absolute viscosity of fluid film and applied load with respect to the upper and the lower limits for the ellipticity parameter 


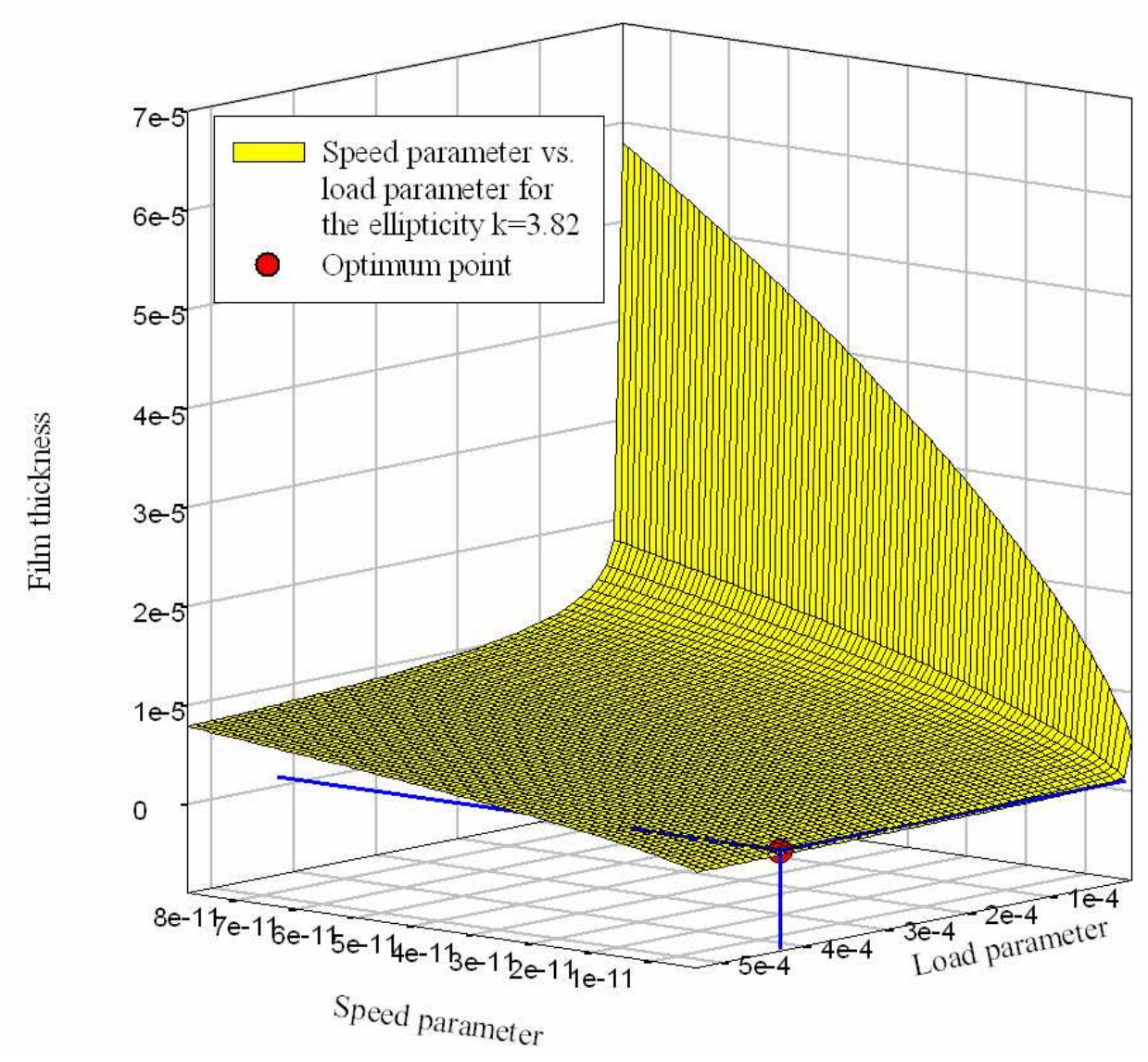

Figure 4 The objective function values versus the absolute viscosity of fluid film and the applied load for the optimum value of the obtained optimum ellipticity parameter $(K=3.82)$

\section{CONCLUSION}

Fluid film thickness will persist as an active area of study for the foreseeable future because of its fundamental importance in the design of implant in human body. This study employs a nature inspired algorithm, the SA, to find minimum fluid film thickness in artificial human knee joint. It is important to optimize the film thickness of the bearing in terms of successful operation and lifetime of the implant in terms of friction and wear. The SA is derived from analogy with natural system behavior and start with an initial random point. It comprises three operations- generation, acceptance, and cooling to produce a new generation. The SA deal with optimization problems that have numerous locally optimum solutions. Standard nonlinear programming techniques to solve optimization problems, in most cases, find a local optimum that is closest to the starting point. In contrast, the SA is well studied to find the global optimum with a high probability as in this study. The major disadvantage of the SA is computation intensive. It can be concluded that nature inspired algorithm is proven to be robust and has demonstrated its capability to produce an efficient solution. 


\section{REFERENCES}

1. V. Wright and D. Dowson, Lubrication and cartilage, J.Anat., 121, 1, 107-118, 1976. 2. A. Unsworth, D. Dowson, and V. Wright, Some new evidence on human joint lubrication, Ann. Rheum. Dis. 34, 277-285, 1975.

3. S. Kirkpatric, C.D. Gelatt, and M.P. Vecchi, Optimization by simulated annealing, Science, 220, 671-680, 1983.

4. N. Metropolis, A. Rosenbluth, M. Rosenbluth, A. Teller, and E. Teller, (1953), Equation of state calculations by fast computing machines, J. Chem. Phys. 21, 10871090, 1953.

5. A. Corana, M. Marchesi, C. Martini, S. Ridella, Minimizing multimodal function of continuous variables with the simulated annealing algorithm, ACM Trans. On Mathematical Software, 13, 11, 87-100, 1992.

6. M. Miki, T. Hiroyasu, and O. Keiko, Simulated annealing with advanced adaptive neighborhood, Journal of information processing society of Japan, 44, 1, 1-6, 2003.

7. M. Miki, S. Hiwa, and T. Hiroyasu, Simulated annealing using adaptive search vector, IEEE, 2006.

8. E.L. Radin and I.L. Paul, A Consolidated concept of joint lubrication. J Bone Joint Surg Am, 54, 607-616, 1972.

9. D.V. Davis and J. Palfrey, Physical properties of synovial fluid. Lubrication and Wear in Joints, 20-28, 1969.

10. R. Vos and F. Theyse, Lubricating properties of synovial fluid in human and animal joints. Lubrication and Wear in Joints, 29-34, 1969.

11. J.B. Lou, K.Y. Lawrence, B. Shi, and S.Z. Wen, Thin film lubrication and lubrication map. Twenty-sixth leeds-lyon symposium on tribology, Leeds, 1999.

12. J.B. Hamrock and D. Dowson, Lubrication background, NASA Technical Memorandum 81692, 1981.

13. J.B. Hamrock and D. Dowson, Elastohydrodynamic lubrication of elliptical contacts for materials of low elastic modulus I- Fully flooded conjunction, NASA TN D-8528 Report, 1977.

14. J.B. Hamrock and D. Dowson, Isothermal elastohydrodynamic lubrication of point contacts. Part I- Theoretical formulation, J.Lubr.Technol, 98, 2, 223-229, 1976.

15. D.J. Hamrock, S.R. Schmid, and B.O. Jacobson, Fundamentals of Fluid Film Lubrication, CRC press, 2004.

16. A.J. Smith, A study of forces in the body in athletic activities with particular reference to jumping. Ph.D.Thesis, University of Leeds, 1972.

17. A. Homaifar, C.X. Qi, and S.H. Lai, Constrained optimization via genetic algorithms", Simulation, 62, 242-254, 1994. 